\title{
Estudio sobre la intuición artificial
}

Octavio Díaz Hernández

\section{Resumen}

En este trabajo se exponen los conceptos y las propiedades de la intuición artificial que se han generado por medio de un análisis de fuentes de información y experimentación propia. Asimismo, se plantea una clasificación para los algoritmos que integran el procesamiento de resolución de problemas a través de la intuición artificial. En este artículo se da a conocer un modelo innovador para que se desarrollen nuevos algoritmos en control de máquinas, donde la intuición artificial es el pilar para que el procesamiento de la información se realice con mayor rapidez y sus requerimientos de procesamiento sean mínimos.

Palabras clave: intuición artificial, algoritmos, modelo, robótica, programación.

\section{A STUDY ABOUt ARTIFICIAL INTUITION}

\begin{abstract}
In this paper we discuss concepts and properties of artificial intuition, synthetized from the analysis of literature and our own experimentation. Also, we report an algorithms' classification, which embodies artificial intuition in machines for problem solving. This article establishes an innovative model for the development of new algorithms in machine control, where artificial intuition allows a faster information processing and minimum processing requirements.
\end{abstract}

Keywords: artificial intuition, algorithms, model, robotics, programming. 
Cursó la Licenciatura en Ingeniería Mecatrónica, en la Facultad de Ingeniería de la Universidad Nacional Autónoma de México (UNAM). Obtuvo el grado de maestro, con mención honorífica, y el grado de doctor en la División de Estudios de Posgrado de la Facultad de Ingeniería de la UNAM. Fue profesor en el departamento de mecatrónica de su facultad por nueve años. Ha impartido las asignaturas de Electrónica analógica y digital, Diseño mecatrónico y Automatización industrial, a nivel licenciatura. Ha participado en proyectos institucionales y con vinculación a la industria. Participó en la creación de la Licenciatura en Ingeniería en Sistemas Biomédicos. Sus líneas de investigación se han enfocado a la robótica serial, paralela y móvil. Ha publicado artículos en congresos y revistas nacionales e internacionales. Ha dirigido tesis en las áreas de ingeniería mecatrónica y ha participado como sinodal en múltiples exámenes profesionales a nivel licenciatura y exámenes de grado a nivel maestría. Es miembro del Instituto de ingenieros eléctricos y electrónicos (Institute of Electrical and Electronics Engineers, IEEE) y de la Sociedad Mexicana de Ingeniería Mecánica, somim.

Actualmente es profesor de tiempo completo en la Facultad de Estudios Superiores Aragón en el departamento de Ingeniería Eléctrica Electrónica y está proponiendo proyectos sobre rehabilitación aplicando ingeniería mecatrónica.

\section{Introducción}

La intuición humana es de interés para los que estudian el arte de generar algoritmos para máquinas, robots u otros entes artificiales. En la actualidad, el tema de la intuición se ha ido derivando desde el campo de la especulación filosófica hacia el campo de la ciencia positiva, siendo considerado más bien un tema de investigación psicológica y neurológica. La palabra intuición proviene del latín intueri, que significa "a considerar" y se puede entender como un tipo de percepción, pero además como un conocimiento implícito de la realidad en la mente inconsciente de los seres humanos. Los mecanismos de intuición realizan un reconocimiento de símbolos o señales que es llevado a cabo sin el procesamiento consciente o racional del individuo. Después del reconocimiento, automáticamente se sintetizan juicios, pensamientos, decisiones o acciones de acuerdo con la información procesada. Este tipo de juicios son caracterizados por ser apropiados, certeros, coherentes, rápidos e incluso evidentes (Jung, 1953; Ferrater-Mora, 1984; Krippendorff, 1986; Hogarth, 2001; Hogarth, 2002; Kahneman, 2003; Simon y Frantz, 2003; Goldstein, 2005; Kant, 2005; Erlhoff, Marshall et al., 2008; Harteis, Koch et al., 2008; Seligman y Kahana, 2009; Kahneman, 2012; Morsella y Poehlman, 2013).

En este trabajo se modela la intuición artificial como punto de partida para una línea de investigación naciente, la cual busca la programación de una "mente artificial" más eficiente, para lo que la intuición artificial se diseña y se programa en forma de algoritmos de bajo gasto computacional, que podrán resolver tanto problemas elementales como complejos. 


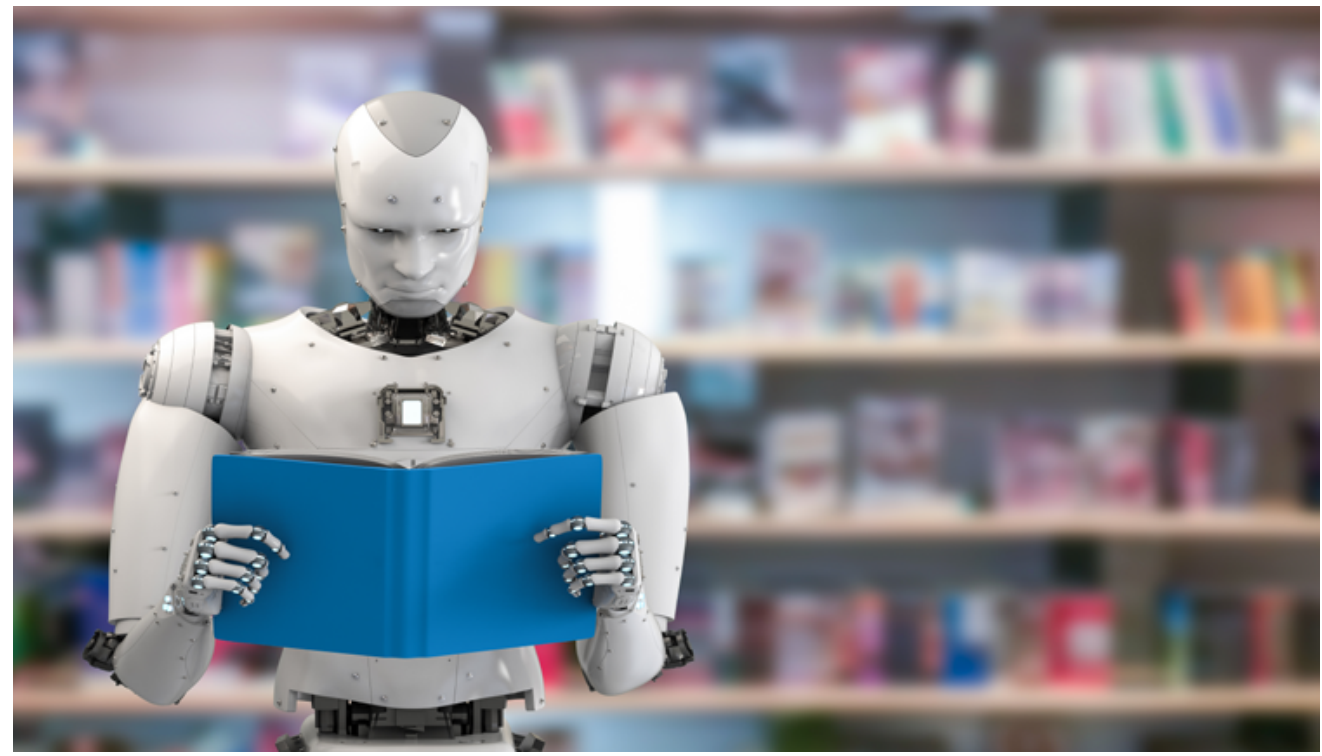

Para comprender mejor la intuición artificial, es necesario describir la fuente en la que se inspiró: la intuición humana. La mente humana es la responsable del entendimiento, capacidad de crear pensamientos, creatividad, aprendizaje, raciocinio, percepción, emoción, memoria, imaginación, voluntad, intuición, y otras habilidades cognitivas (Sevilla y Casacuberta, 2001; Van-Gulick, 2014). Desde una perspectiva cognitiva, se considera que la mente funciona por dos vías: a) la mente consciente o explícita, discursiva, secuencial, racional, y que requiere un esfuerzo para que funcione. $Y$, por otro lado, existe una mente inconsciente u oculta, implícita, asociativa, rápida y que no requiere de esfuerzo (Kahneman, 2003; Myers, 2008). De ahí que, en situaciones complejas, en las que se manejan grandes cantidades de información, las elecciones realizadas mediante pensamiento inconsciente pueden resultar incluso más eficaces que las mediadas por deliberaciones conscientes (Dijksterhuis, Nordgren et al., 2006 ). En la figura 1 se muestra una representación de la calidad de las decisiones con relación a los pensamientos conscientes e inconscientes.

Figura 1. Relación entre complejidad y calidad de una decisión, según se afronte mediante el pensamiento consciente 0 inconsciente (Dijksterhuis, Nordgren et al., 2006).

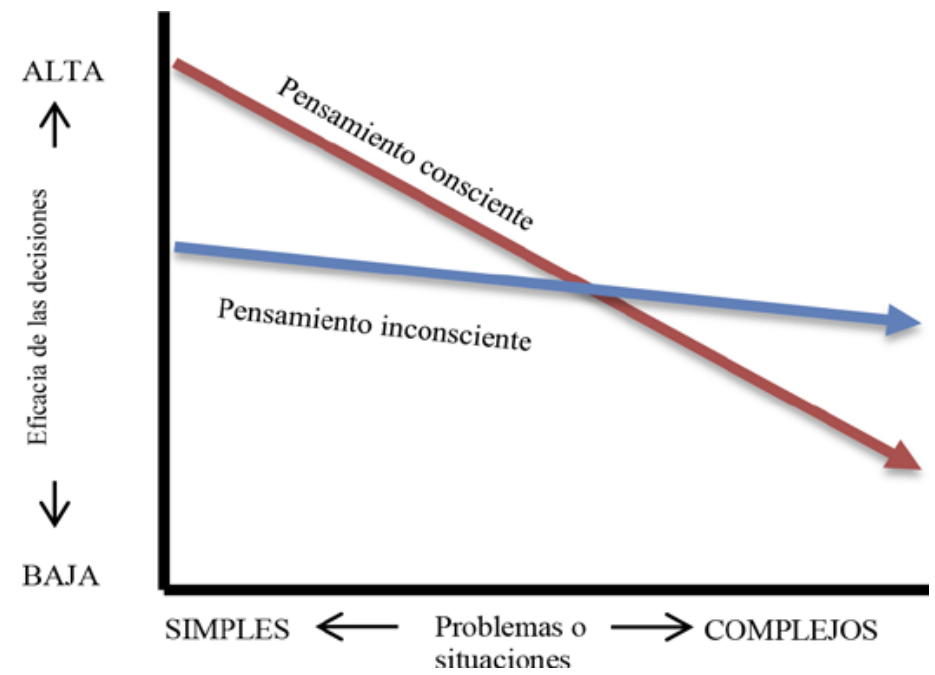


Figura 2. Proporcionalidad de los procesos conscientes e inconscientes en la mente humana.
El inconsciente libera a la consciencia y se ocupa de las cosas en forma automatizada, mientras que el consciente puede ser contradictorio. El inconsciente es un proceso primario y el consciente es un proceso secundario donde están contenidas las funciones cognitivas como la inteligencia, aprendizaje, concentración, entre otras (Hogarth, 2001; Hassin, Uleman et al., 2005; Froufe, Sierra et al., 2009; Morsella y Poehlman, 2013; Van-Gulick, 2014). En la figura 2 se muestra la proporcionalidad de los procesos mentales que son conscientes e inconscientes, y se hace énfasis en que los pensamientos inconscientes sostienen a los pensamientos conscientes. Asimismo, la intuición es un proceso mental de tipo inconsciente.

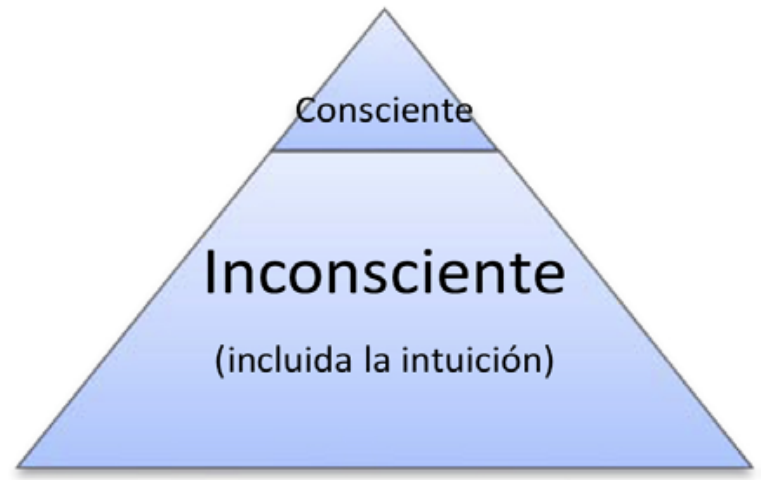

El término consciente artificial, normalmente aplicado a robots, alude a que la programación debe controlar acciones características de la "voluntad propia", siempre en camino de hacer robots eficientes y que muestren "autonomía" (MacLennan, 2005). El problema de la consciencia en robots implica que reflejen ser astutos y autoreflexivo. Lo anterior ha resultado en la complejidad de los algoritmos de consciencia artificial (Molyneux, 2012). Para la formulación e implementación del consciente en máquinas la comunidad científica ha buscado por décadas el lado racional de las habilidades humanas, planteado así la inteligencia artificial —se invita a estudiar su origen y desarrollo en las referencias (Russell y Norvig, 2003; Martínez y Britos, 2004; Anderson, 2007)—. Por otro lado, existe el término inconsciente artificial, que, históricamente, se ha manifestado en la llamada automatización, o mecanización de los procesos. La automatización incluye pero no limita la implementación de acciones repetitivas, predecibles y preprogramadas en un dispositivo, proceso o sistema para que se ejecuten tareas disminuyendo o eliminando la intervención humana, cabe resaltar que en la automatización no hay condiciones ni respuestas aleatorias (Gupta y Arora, 2007). También existen algunos trabajos que hablan de elementos "vestigiales" de la mente humana más útiles que el raciocinio para el control de robots (Brooks, 1991a). Por ejemplo, está la programación de comportamientos reactivos en robots (Arkin, 1998) y las emociones en robots, las cuales podrían considerarse como una aproximación de la implementación del inconsciente artificial; aunque aún no se hace evidente esta relación, propongo clasificarlas de este modo luego de un análisis de la literatura correspondiente (Minsky, 2006; Velasquez, 2007; Yang, Malaka et al., 2010). No obstante, es importante resaltar que la intuición no debe confundirse con las emociones. 
Hasta este momento, la intuición artificial ha sido trabajada desde el punto de vista computacional (Anderson, 2007; Weidong y Ping, 2009; Dundas y Chik, 2013); sin embargo, la intuición artificial necesita ser estudiada desde sus fundamentos como una teoría transdisciplinaria.

\section{La intuición aplicada a sistemas artificiales en la actualidad}

En años recientes la intuición ha llamado la atención para el mejoramiento del desempeño de las máquinas, teniendo como ejemplo algunos algoritmos computacionales (Gobet y Chassy, 2009). Ese es el caso de Mónica Anderson, quien se ha interesado por la cognición basada en computación y se ha dedicado a buscar alternativas para la inteligencia artificial, lo que denomina intuición artificial, la cual describe como métodos subsimbólicos de bajo gasto computacional que pueden resolver problemas más elementales, y en conjunto podrían resolver los problemas actuales de la inteligencia artificial, por ejemplo el bootstrapping1, que requiere alto poder de procesamiento para ejecutar comandos de alto nivel, como el reconocimiento de voz o de rostros (Anderson, 2007). También la intuición artificial se ha planteado como el diseño de software cooperativo entre programadores y usuarios (Weidong y Ping, 2009), además de que otros investigadores insinúan que puede verse simplistamente como un reconocimiento de patrones usando técnicas convencionales de inteligencia artificial (Dundas y Chik, 2013).

\section{Modelo de intuición artificial}

A continuación, se aportan al conocimiento algunos conceptos y propiedades de la intuición artificial (InA). Cabe aclarar que los siguientes párrafos son el resultado de una investigación propia a través del análisis de la literatura sobre la intuición humana y otros temas sobre la mente, robótica, inteligencia artificial, entre otros.

\section{Definiciones de la intuición artificial}

a) La ınA es un área multidisciplinaria que, a través de las ciencias, tales como informática, psicología y robótica, estudia la creación y diseño de entidades capaces de emular ciertos rasgos de la intuición humana.

b) La InA es una rama de la inteligencia artificial, que se dedica a emular el reconocimiento automático de patrones de información para generar respuestas rápidas y normalmente certeras o confiables.

1 En general, el llamado bootstrapping en programación se refiere a un proceso que puede autoiniciarse sin entradas externas. c) La InA es una representación limitada de las capacidades intuitivas del ser humano, programadas en un ente artificial.

d) La InA es un procesamiento basado en el reconocimiento automático de patrones, lo que la hace extremadamente rápida y no hace uso exhaustivo de la capacidad de memoria ni de procesamiento en la búsqueda razonada o iterativa de soluciones. 


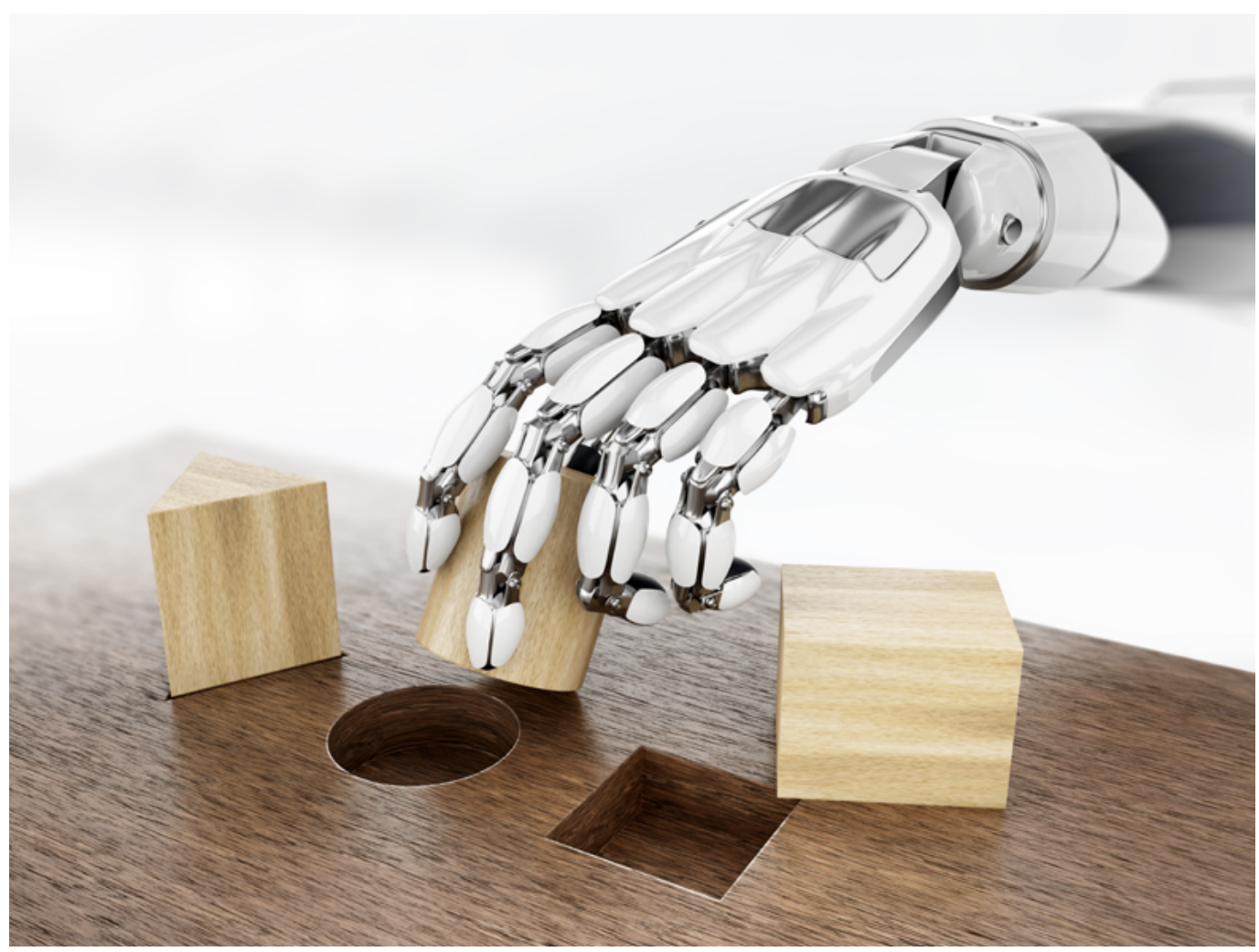

\section{Propiedades de la intuición artificial}

a) La ina emula la vía intuitiva de la obtención de soluciones que no necesitaron un largo proceso de búsqueda, en su lugar hubo un reconocimiento de fragmentos relevantes de información que conllevan al descubrimiento de una solución que mejorará la calidad de las respuestas.

b) La ınA funciona con base en un conjunto de algoritmos sintetizados a partir del estudio de las capacidades humanas intuitivas para realizar una tarea en condiciones que estimularon la intuición. Estos algoritmos representan mecanismos inconscientes compuestos por fragmentos o plantillas de conocimiento preadquirido. Las funciones de cada algoritmo trabajan de manera independiente o se presentan bajo el principio de superposición.

c) La ina y sus algoritmos están embebidos en los elementos de procesamiento del agente artificial como parte de su arquitectura.

d) La ina se implementa con ecuaciones o fórmulas, cuyas entradas son piezas relevantes de información para la tarea, lo que cumple con la propiedad de un procesamiento automático.

e) La ina encuentra la respuesta a un problema en cuanto recibe las entradas, discriminando otras posibles soluciones; en consecuencia, se disminuye el tiempo de búsqueda y la cantidad de procesamiento. En la figura 3 se esquematiza el proceso intuitivo para la resolución de un problema (localizado en la parte superior) en comparación con un proceso racional (localizado en la parte inferior). 


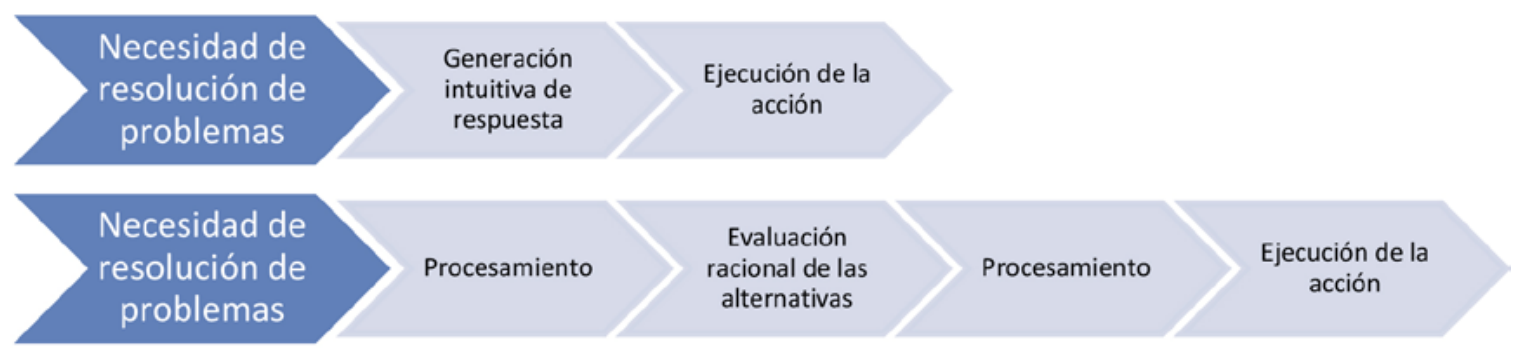

Figura 3. Comparación de procesos de resolución de problemas. Abajo, con cinco etapas, se muestra el proceso convencional, en relación a un proceso realizado con intuición artificial que se muestra arriba, con tres etapas.

\section{Clasificación de la intuición artificial}

Así como las definiciones y propiedades de la ınA, en este artículo se clasifican los algoritmos de ina en dos tipos, los cuales serán programados en un ente artificial y pueden funcionar simultáneamente:

1. Algoritmos de intuición artificial primitiva (InAP). Se reconoce como primitivo a aquel algoritmo que se considera una estructura elemental y necesaria para la continuidad y preservación del agente artificial. Los algoritmos de inAp representan el conjunto de atajos heurísticos desarrollados a lo largo de la evolución.

2. Algoritmos de intuición artificial adquirida (InAA). Se le llama adquirido al algoritmo que fue definido a partir de la tarea por ejecutar. Se asocia con la repetición de eventos y puede estar inspirado en la intuición humana para ejecutar tareas. Los algoritmos de InAA representan a las asociaciones aprendidas a lo largo de la vida.

Es importante recalcar que la intuición artificial no es aprendida de forma autónoma por el ente artificial, ya que esto implicaría romper con el esquema de un procesamiento rápido y de bajo costo computacional. La razón principal es que se recurriría a herramientas como adquisición y procesamiento de señales de entrada —como lenguaje natural (ej. habla humana) o visión artificial_-, los cuales son una característica fundamental de los entes racionales (inteligencia artificial). Por lo que en este modelo de intuición artificial no se contempla el aprendizaje de acciones intuitivas, a partir de las entradas al sistema, por el propio sistema artificial. Por lo tanto, estos algoritmos son programados por una persona de forma "bioinspirada".

\section{Conclusiones}

La intuición artificial está inspirada en el mecanismo de la mente intuitiva humana inconsciente, que posee ventajas como el procesamiento automático de gran cantidad de información, en múltiples dimensiones y sin el decaimiento de la eficacia ante situaciones complejas. La intuición artificial emula la vía intuitiva de la obtención de soluciones que no necesitaron un largo proceso de búsqueda, en su lugar hubo reconocimiento de fragmentos relevantes de información que conllevan al descubrimiento de una solución que mejorará la calidad de las respuestas. 
En la intuición artificial influyen dos tipos de algoritmos: por un lado, están los de intuición artificial primitiva, que están diseñados por un programador a partir de las intuiciones humanas, que son las que capacitan a los seres humanos para los juicios rápidos y frugales. Por otro lado, están los algoritmos de intuición artificial adquirida, los cuales también son programados por una persona, pero que toma como inspiración ciertas asociaciones aprendidas gracias a la práctica o experiencia personal de un individuo o de un grupo de personas, obteniéndose un promedio.

Los sistemas actuales de inteligencia artificial sólo han contemplado los elementos racionales, como la inteligencia artificial convencional, redes neuronales y agentes autónomos de aprendizaje, que reconocen patrones o ejercen tareas con medios informáticos orientados a emular la consciencia humana. A partir de este trabajo se clasifica en dos ramas la inteligencia artificial: 1) sistemas de consciencia artificial y 2) sistemas de inconsciencia artificial. Con base en estos últimos se pueden obtener sistemas robóticos semiautónomos y autónomos con una toma de decisiones rápida y acertada.

Debido a que la extensión de este trabajo es limitada, se invita al lector a profundizar en el tema de la intuición humana:

\section{Intuición artificial aplicada al a teleoperación.}

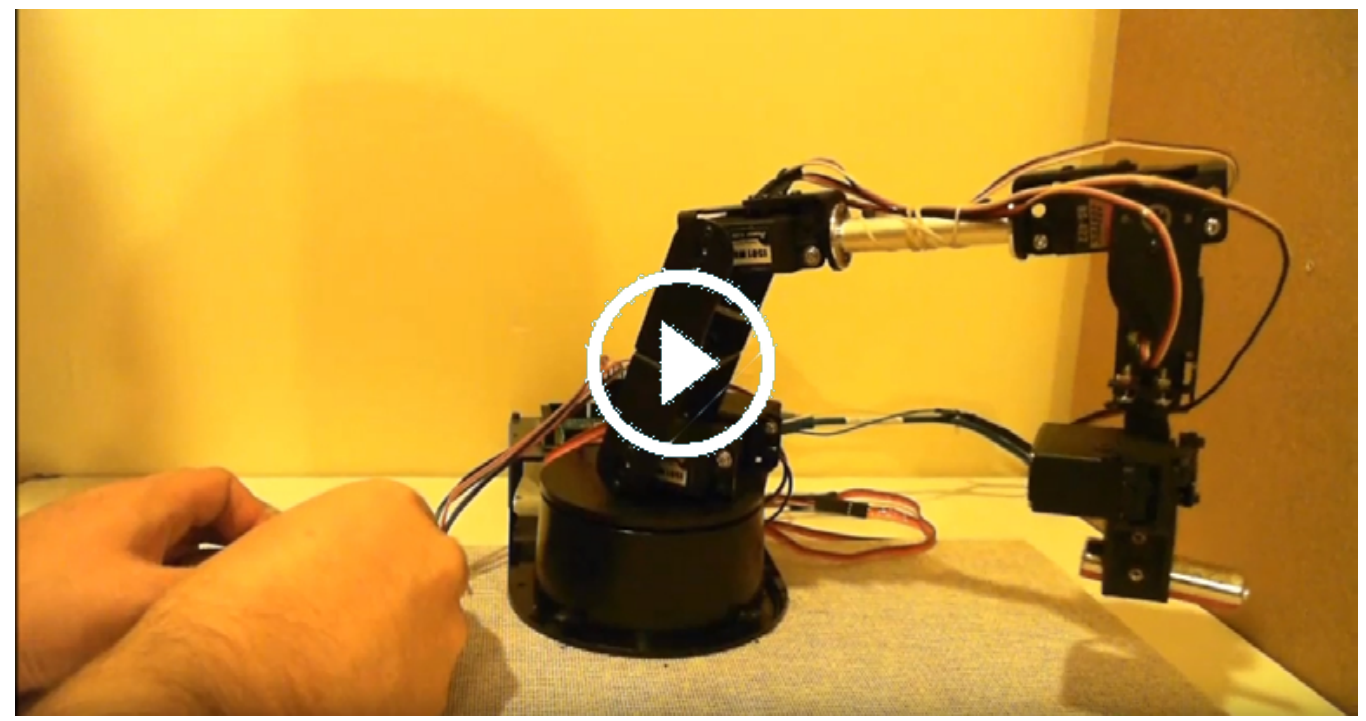

\section{Estudios antecedentes sobre la intuición humana}

Chase y Simon, 1973; Dreyfus y Dreyfus, 1988; Easen y Wilcockson, 1996; Hogarth, 2001; Hogarth, 2002; Kahneman, 2003; Simon y Frantz, 2003; Gladwell, 2005; Kant, 2005; Seligman y Kahana, 2009; Kruglanski y Gigerenzer, 2011; Isenman, 2013; Marcovici y Blume-Marcovici, 2013; Pearson, 2013; Tinghög, Andersson et al., 2013; Woolley y Kostopoulou, 2013). 


\section{La intuición humana y sus propiedades}

Chase y Simon, 1973; Dreyfus y Dreyfus, 1988; Simon, 1992; Easen y Wilcockson, 1996; Hogarth, 2001; Hogarth, 2002; Kahneman, 2003; Simon y Frantz, 2003; Gladwell, 2005; Minsky, 2006; Seligman y Kahana, 2009; Kruglanski y Gigerenzer, 2011; Kahneman, 2012; Isenman, 2013; Marcovici y Blume-Marcovici, 2013; Pearson, 2013; Tinghög, Andersson et al., 2013; Woolley y Kostopoulou, 2013).

\section{Referencias}

* Anderson, M. (2007). Artificial Intuition: A New Possible Path to Artificial Intelligence. Recuperado de: http://artificial-intuition.com/anderson.html.

Arkin, R. C. (1998). Behavior-Based Robotics. Cambridge, MA, USA: The MIT Press.

- Brooks, R. A. (1991a). Intelligence without Reason. Proceedings of 12th Int. Joint Conf. on Artificial Intelligence, Sydney, Australia.

* Chase, W. G. y H. A. Simon (1973). The mind's eye in chess. Visual information processing (New York: Academic Press): 215-281.

* Diaz-Hernandez, O. (2014). Intuición Artificial Aplicada a la Teleoperación. Doctorado en ingeniería, campo disciplinario Mecatrónica. Ciudad de México, Universidad Nacional Autónoma de México. Doctorado.

* Dijksterhuis, A., L. F. Nordgren, et al. (2006). On making the right choice: the deliberation-without-attention effect. Science, 311(5763): 1005-7.

- Dreyfus, H. L.yS. E. Dreyfus (1988). Mind over machine: The power of human intuition and expertise in the era of the computer. New York: Free Press.

- Dundas, J. y D. Chik (2013). Machine implementation of human-like intuition mechanism in Artificial Intelligence. ICIC Express Letters, 7(8): 2231-2235.

- Easen, P. y J. Wilcockson (1996). Intuition and rational decision-making in professional thinking: a false dichotomy? Journal of Advanced Nursing, 24(4): 667673.

- Erlhoff, M., T. Marshall, et al. (2008). Intuition. Design Dictionary. (s. n.): Birkhuser Basel, pp. 236-236.

* Ferrater-Mora, J. (1984). Diccionario de Filosofía (4 tomos). A. Diccionarios: Barcelona.

* Froufe, M., B. Sierra, et al. (2009 ). El inconsciente Cognitivo en la psicología científica del S. XXI. Recuperado de: https://www.u-cursos.cl/facso/2011/1/PSIMTCCC/1/material docente/bajar?id material=576906.

Gladwell, M. (2005). Blink, Inteligencia Intuitiva. Taurus.

* Gobet, F. y P. Chassy (2009). Expertise and Intuition: A Tale of Three Theories. Minds \& Machines, 19: 151-180. 
* Goldstein, E. B. (2005). Cognitive psychology: Connecting mind, research, and everyday experience. Australia Belmont: Thomson/Wadsworth.

- Gupta, A. K. y S. K. Arora (2007). Industrial Automation and Robotics. Laxmi publications.

- Harteis, C., T. Koch, et al. (2008). How intuition contributes to high performance: An educational perspective. us-China Education Review, 5(1): 68-80.

* Hassin, R. R., J. S. Uleman, et al. (2005). The new unconscious. Oxford University Press.

* Hogarth, R. (2001). Educating intuition. Chicago: University of Chicago Press.

- Hogarth, R. (2002). Deciding Analytically Or Trusting Your Intuition?: The Advantages and Disadvantages of Analytic and Intuitive Thought. Universitat Pompeu Fabra. Departament d'Economia i, Empresa, Universitat Pompeu Fabra.

* Isenman, L. (2013). Understanding Unconscious Intelligence and Intuition: "Blink" and Beyond. Perspectives in Biology and Medicine, 56(1): 148-166.

* Jung, C. G. (1953). Psychological types, or, The Psychology of individuation. Pantheon.

- Kahneman, D. (2003). A Perspective on Judgment and Choice. American Psychologist, 58(9): 697-720.

* Kahneman, D. (2012). Pensar rápido, pensar despacio. España: Penguin Random House.

- Kant, I. (2005). Crítica de la Razón Pura. Taurus.

- Krippendorff, K. (1986). A Dictionary of Cybernetics. T. A. S. O. Communications. Philadelphia PA 19104, USA: University of Pennsylvania.

* Kruglanski, A. W. y G. Gigerenzer (2011). Intuitive and Deliberate Judgments Are Based on Common Principles. Psychological Review, 118(1): 97-109.

* Maclennan, B. J. (2005). Consciousness in robots: the hard problem and some less hard problems. Robot and Human Interactive Communication, 2005. ROMAN 2005. IEEE International Workshop on.

- Marcovici, P. y A. Blume-Marcovici (2013). Intuition versus rational thinking: psychological challenges in radiology and a potential solution. Journal of the American College of Radiology, 10(1): 25-29.

* Martínez, R. G. y P. V. Britos (2004). Ingeniería de Sistemas Expertos. Nueva Librería.

Minsky, M. (2006). The Emotion Machine: Commonsense Thinking, Artificial Intelligence, and the Future of the Human Mind. Simon y Schuster.

* Molyneux, B. (2012). How the Problem of Consciousness Could Emerge in Robots. Minds and Machines, 22(4): 277-297.

* Morsella, E. y T. A. Poehlman (2013). The inevitable contrast: Conscious vs. unconscious processes in action control. Front Psychol., 4: 590. 
* Myers, D. G. (2008). El poder y los peligros de la intuición. Mente y Cerebro, 33: 2229.

* Pearson, H. (2013). Science and intuition: do both have a place in clinical decision making? British Journal of Nursing, 22(4): pp 212 - 215.

- Russell, S. J. y P. Norvig (2003). Artificial Intelligence: A Modern Approach. Upper Saddle River, New Jersey, Prentice Hall.

- Seligman, M. E. P.y M. Kahana (2009). Unpacking Intuition: A Conjecture. Perspective Psycholgy Science, 4(4): 399-402.

* Sevilla, D. C. y D. Casacuberta (2001). La mente humana, Oceano Difusion Editorial, S. A.

- Simon, H.y R. Frantz (2003). Artificial intelligence as a framework for understanding intuition. Journal of Economic Psychology, 24: 265-277.

- Simon, H. A. (1992). What Is an "Explanation" of Behavior? Psychological Science, 3(3): 150-161.

* Tinghög, G., D. Andersson, et al. (2013). Intuition and cooperation reconsidered. Nature, 498(E1-E2).

*an-Gulick, R. (2014). Consciousness. The Stanford Encyclopedia of Philosophy. E. N. Zalta.

- Velasquez, D. (2007). When Robots Weep. Electrical enginering and computacional science. Massachusetts, USA, Massachusetts Institute of Technology. PhD: 262.

- Weidong, T. y H. Ping (2009). Intuitive Learning and Artificial Intuition Networks. Second International Conference on Education Technology and Training.

- Woolley, A. y O. Kostopoulou (2013). Clinical intuition in family medicine: more than first impressions. Annals of Familiar Medicine, 11(1): 60-6.

* Yang, H., R. Malaka, et al. (2010). Emotions: The Voice of the Unconscious. Entertainment Computing - ICEC 2010, Springer Berlin Heidelberg. 6243: 205-215.

\section{Cómo citar este artículo}

* Díaz Hernández, Octavio (2019). Estudio sobre la intuición artificial. Revista Digital Universitaria (RDU). Vol. 20, núm. 2 marzo-abril. DOI: http://doi.org/10.22201/ codeic.16076079e.2019.v20n2.a3 